\title{
Da gaveta para a indústria: correspondência entre patentes universitárias e atividades econômicas ${ }^{1}$
}

\section{Aziz Eduardo Calzolaio}

Professor da Universidade de Caxias do Sul e da Faculdade de Tecnologia do Cooperativismo (ESCOOP), possui Doutorado em Economia pela UFRGS, com período de Doutorado Sanduíche na Scuola Superiore Sant'Anna, Pisa - Itália, Rio Grande do Sul, Brasil aziz-calzolaio@sescooprs.coop.br

Gisele Spricigo

Coordenadora do Curso de Graduação em Ciências Contábeis, Ciências Econômicas e Gestão Financeira da UNISINOS e professora nos cursos de graduação da Escola de Gestão e Negócios da Unisinos, possui Doutorado em Economia pela UFRGS. Rio Grande do Sul, Brasil giseles@unisinos.br.

Sergio Marley Modesto Monteiro

Professor da Universidade Federal do Rio Grande do Sul, possui Doutorado em Economia pela UFRGS. Endereço: Universidade Federal do Rio Grande do Sul, Faculdade de Ciências Econômicas, Rio Grande do Sul, Brasil sergio.monteiro@ufrgs.br.

Editor Científico: José Edson Lara
Organização Comitê Científico
Double Blind Review pelo SEER/OJS
Recebido em 19.09.2017
Aprovado em 14.02.2018

${ }^{1}$ Esse trabalho é parte dos resultados da Bolsa de Pós-Doutorado - DOCFIX - Edital CAPESFAPERGS no 05-2013, da qual Aziz Eduardo Calzolaio foi bolsista de set. de 2015 a out. de 2016. Agradecemos o apoio financeiro da FAPERGS E CAPES.
} 


\section{Resumo}

O objetivo principal desse artigo é o de realizar a correspondência entre as patentes da Universidade Federal do Rio Grande do Sul (UFRGS) e as atividades econômicas da Classificação Nacional das Atividades Econômicas (CNAE). Para tanto, foi utilizada a metodologia denominada Algorithmic Links with Probabilities, que faz uso de uma estrutura probabilística para estabelecer a conexão dos dados de patentes com palavras-chave extraídas das descrições da indústria. Após a utilização da técnica, procedeu-se à Análise de Correspondência e ao teste Qui Quadrado sobre os resultados. Concluiu-se que existe uma relação estatisticamente significativa entre as patentes da UFRGS e atividades econômicas específicas. Observou-se que $76 \%$ das patentes da UFRGS estão relacionadas a somente 6 campos econômicos. As patentes concentram-se no campo médico, odontológico, higiênico e terapêutico e relacionam-se, principalmente, com as indústrias de medicamentos e, secundariamente, com as de defensivos agrícolas e vacinas veterinárias. Complementarmente, as tecnologias de engenharia de material estão associadas às indústrias de máquinas e equipamentos.

Palavras-chave: Patentes; Atividades Econômicas; Transferência Tecnológica; Universidades.

\section{From the drawer to the industry: correspondence between university patents and economic activities}

\section{Abstract}

The main objective of this article is to match the patents from Federal University of Rio Grande do Sul (UFRGS) and the economic activities of the Economic Activities National Classification (CNAE). The matching was performed using the methodology called "Algorithmic Links with Probabilities", which uses a probabilistic framework to establish the connection between the patent data with keywords extracted from the industry descriptions. After the use of the technique, correspondence analysis and a Chi-Square test were performed on the results. The analysis showed that there is a statistically significant relationship between UFRGS patents and specific economic activities. It was observed that $76 \%$ of the patents of UFRGS are related to only 6 economic fields. Patents are concentrated in the medical, dental, hygienic and therapeutic fields and are mainly related to the drug industry and, secondarily, to agricultural pesticides and veterinary vaccines industries. In addition, material engineering technologies are associated with the machinery and equipment industries.

Keywords: Patents; Economic activities; Technology Transfer; Universities. 


\section{Del cajón a la industria: correspondencia entre patentes universitarias y actividades económicas}

\section{Resumen}

El objetivo principal de este artículo es el de realizar la correspondencia entre las patentes de la Universidad Federal do Rio Grande do Sul (UFRGS) y las actividades económicas de la Clasificación Nacional de las Actividades Económicas (CNAE). Para eso, se utilizó la metodología denominada Algorithmic Links with Probabilities, que hace uso de una estructura probabilística para establecer la conexión de los datos de patentes con palabras claves extraídas de las descripciones de la industria. Después de la utilización de la técnica, se procedió al Análisis de Correspondencia y a la prueba de Chi Cuadrado sobre los resultados. Se concluyó que existe una relación estadísticamente significativa entre las patentes de la UFRGS y actividades económicas específicas. Se observó que el $76 \%$ de las patentes de la UFRGS están relacionadas solamente a 6 campos económicos. Las patentes se concentran en el campo médico, odontológico, higiénico y terapéutico y se relacionan, principalmente, con las industrias de medicamentos y secundariamente, con las de agroquímicos y vacunas veterinarias. Complementariamente, las tecnologías de ingeniería de material están asociadas a las industrias de máquinas y equipamientos.

Palabras clave: Patentes; Actividades Económicas, Transferencia Tecnológica; Universidades.

\section{Introdução}

As instituições de ensino e pesquisa habilitam-se cada vez mais para colaborarem com outras organizações não acadêmicas com o intuito de gerarem conhecimentos úteis ao setor produtivo (Perkmann et al., 2013). Por isso, a transferência tecnológica constitui-se como uma nova missão das universidades (Kalar \& Antoncic, 2015). Porém, pouco se sabe a respeito dos mecanismos subjacentes à transferência tecnológica (Buenstorf \& Geissler, 2012). Existe, portanto, uma carência de pesquisas que investiguem a operacionalização da transferência de uma tecnologia originada em uma universidade (Desidério \& Zilber, 2014).

Houve um aumento da comercialização dos resultados das pesquisas acadêmicas (Mueller \& Perucchi, 2014) desde a criação da Lei № 10.973/2004, chamada de Lei da Inovação. Esta Lei permitiu de forma clara a comercialização dos 
resultados de pesquisas advindas das Instituições de Ciência e Tecnologia (ICT) ${ }^{2}$ brasileiras. A política de inovação, adotada desde 2004, gerou incentivos para a implementação dos Núcleos de Inovação Tecnológica (NITs) nas instituições de ensino superior (Rosa \& Frega, 2017), com o objetivo de incentivar e promover tanto a proteção intelectual como a comercialização das tecnologias de maneira eficiente (Castro \& Souza, 2012). Ademais, a importância das descobertas científicas para a inovação das empresas conduziu os gestores de patentes ${ }^{3}$ a visualizarem-nas como ativos com potencial econômico.

Por um lado, novas metodologias prospectam mercado para invenções particulares das ICT em uma perspectiva microeconômica, a exemplo do que fizeram Marques, Pereira, Garcia e Gava (2017) para uma vacina desenvolvida na Universidade Federal de Viçosa. Os autores verificaram a existência de 37 concorrentes, em nível mundial, para a referida tecnologia. Além disso, analisaram a localização, a estrutura de mercado, o faturamento e o nível de atividade de inovação das multinacionais que seriam potenciais parceiros comerciais absorvedores da inovação em questão. Por outro lado, falta uma metodologia que possibilite a visualização de aplicação de todas as patentes que uma universidade possui, já que a venda de uma delas não resolve o problema do "engavetamento" da grande maioria (Desidério \& Zilber, 2014).

Este artigo propõe o uso de uma ferramenta hábil para identificar as possíveis atividades econômicas que têm mais chances do ponto de vista técnico de absorverem determinada tecnologia. Com base nisso, os estudos de inteligência competitiva para transferência de tecnologia seriam direcionados de forma mais eficaz para os mercados com maior potencial de efetivamente avançarem em uma parceria. Desta forma, a relevância deste artigo é oferecer à gestão tecnológica das universidades uma maneira de concentrar-se em setores econômicos mais aptos a desenvolverem negócios.

\footnotetext{
${ }^{2}$ Instituição Científica, Tecnológica e de Inovação (ICT) é definida pela Lei no 13.243/2016, em seu Artigo $2^{\circ}$, Inciso $\mathrm{V}$, como "órgão ou entidade da administração pública direta ou indireta ou pessoa jurídica de direito privado sem fins lucrativos legalmente constituída sob as leis brasileiras, com sede e foro no País, que inclua em sua missão institucional ou em seu objetivo social ou estatutário a pesquisa básica ou aplicada de caráter científico ou tecnológico ou o desenvolvimento de novos produtos, serviços ou processos".

${ }^{3}$ Patentes são definidas como títulos de propriedade temporária sobre uma invenção ou modelo de utilidade que atenda ao requisito de novidade. Elas concedem aos seus detentores um direito exclusivo, sob a tutela do Estado, de explorá-las. (Instituto Nacional de Propriedade Intelectual, 2016)
} 
O Ranking Universitário da Folha (2016) aponta a Universidade Federal do Rio Grande do Sul (UFRGS) como a quinta melhor universidade do país, de acordo com um indicador composto pelos seguintes aspectos: pesquisa, ensino, mercado, internacionalização e inovação (os dados da pesquisa referem-se aos anos de 2005 a 2014). Também é a quinta universidade brasileira em termos de solicitação de patentes ${ }^{4}$. O principal objetivo desse artigo é realizar a correspondência entre os códigos da International Pantent Classification (IPC), utilizados nas patentes da Universidade Federal do Rio Grande do Sul (UFRGS), e as atividades econômicas da Classificação Nacional das Atividades Econômicas (CNAE). Tal desafio é tratado de forma exploratória, visto que este é o primeiro trabalho no Brasil que faz a conexão de um conjunto grande de patentes às atividades econômicas. Essa ligação foi feita com base na proposta de Lybbert e Zolas (2014) e foram utilizadas duas ferramentas estatísticas, a análise de correspondência e um teste Qui Quadrado, para validação dos resultados.

A contar desta introdução, o artigo está organizado em cinco seções. A seção 2 revisa a literatura sobre transferência tecnológica e a correspondência entre patentes e atividades econômicas. A seção 3 apresenta a metodologia utilizada para fazer essa correspondência, bem como as ferramentas estatísticas aplicadas. A seção 4 apresenta e discute os resultados, e a seção 5 contém as considerações finais.

\section{Revisão da Literatura}

\subsection{Transferência tecnológica e a correspondência entre patentes e atividades econômicas}

Nas últimas décadas tem havido um crescimento da comercialização dos resultados das pesquisas acadêmicas em vários países (Thursby \& Gupta, 2007, Organisation for Economic Co-operation and Development, 2009, Mueller \& Perucchi, 2014). Para a operacionalização dessas ações, as invenções acadêmicas

\footnotetext{
${ }^{4}$ As quatro universidades que mais solicitaram patentes foram a Universidade de São Paulo, a Universidade Estadual de Campinas, a Universidade Federal de Minas Gerais e Universidade Federal do Paraná (Ranking Universitário da Folha, 2016).
} 
começaram a ser protegidas de forma mais intensa (Pereira \& Melo, 2015), criandose um ativo para comercialização junto a outras organizações. Desta forma, as ICT constituem-se em uma fonte de receita (Mueller \& Perucchi, 2014), com remunerações através dos royalties.

Após a implementação da Lei da Inovação (Lei no 10.973/2004) no Brasil, houve uma aceleração do crescimento do registro de propriedade intelectual nas universidades brasileiras, o qual permanecia baixo até meados da década de 2000 (Oliveira \& Nunes, 2009). Neste contexto, foi introduzida a permissão da comercialização dos resultados da investigação dos grupos de pesquisas. A partir desse novo arcabouço legislativo, políticas públicas encorajaram a criação dos Núcleos de Inovação Tecnológica, estruturas que realizam a gestão da política institucional de inovação das Instituições de Ciência e Tecnologia (Rosa \& Frega, 2017). Os núcleos estimulam e formalizam as proteções das criações desenvolvidas naquelas instituições, bem como mediam as ações de transferência tecnológica. Já a Lei n 13.243/2016 buscou intensificar a transferência tecnológica com exploração comercial por parte das universidades.

Todavia, na prática, diversos impedimentos restringem a interação universidade-empresa (Nelson, 2006; Apax Partners, 2005). Os relatados por Desidério e Zilber (2014) referem-se às dificuldades de viabilizar uma metodologia que precifique a tecnologia para uma negociação mercadológica. Os autores também mencionam a falta de gestão eficaz de negócios para a transferência tecnológica. Neste sentido, poucas inovações realmente são transferidas das universidades para as empresas (Ismail, Nor, \& Sidek, 2015) e, assim, as patentes continuam "engavetadas". Observa-se que falta uma metodologia que possibilite a identificação das possíveis atividades econômicas que têm chances técnicas de absorverem as patentes.

A realização de uma correspondência entre a IPC e a CNAE pode ajudar nesse processo, mas é algo complexo, visto que tais classificações possuem finalidades diferentes, sem que exista uma conexão direta entre elas. Por um lado, a CNAE ordena os produtos e serviços produzidos no Brasil para atender a finalidades econômicas, como a mensuração da taxa de emprego e do Produto Interno Bruto. Por outro lado, o sistema IPC serve para facilitar o processo de estruturação das áreas científicas atreladas às patentes, auxiliando os examinadores de patentes a 
identificarem com precisão as novas características técnicas da invenção (Lybbert \& Zolas, 2014).

A Tabela 1 apresenta estudos que realizaram as correspondências entre as patentes e as atividades econômicas que foram realizadas até a década de 2010. Essas associações foram realizadas em diferentes países, considerando diversas classificações, tanto de patentes, quanto econômicas

\section{Tabela 1}

Propostas de correspondência entre tecnologias e atividades econômicas

\begin{tabular}{|c|c|c|c|c|}
\hline Autores & Ano & Local ou nome da & Classif & ações utilizadas: \\
\hline & & & Patente & Econômica \\
\hline Schmmokler & 1966 & EUA & $\begin{array}{l}\text { United States } \\
\text { patent class } \\
\text { (USPC) }\end{array}$ & 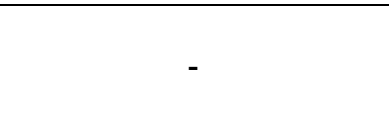 \\
\hline $\begin{array}{l}\text { Escritório de } \\
\text { patente dos EUA }\end{array}$ & $\ldots$ & EUA & ( & - \\
\hline Kronz & 1980 & - & - & - \\
\hline Greif e Potkowik & 1990 & - & - & - \\
\hline $\begin{array}{l}\text { Verspargen, } \\
\text { Morgastel } \\
\text { Slabbers }\end{array}$ & 1994 & $\begin{array}{l}\text { Holanda/Merit } \\
\text { Concordance }\end{array}$ & IPC & $\begin{array}{l}\text { International Standard } \\
\text { Industrial Classification } \\
\text { (ISIC) }\end{array}$ \\
\hline Kortum e Putnam & 1997 & $\begin{array}{l}\text { Canadá/Yale } \\
\text { Technology } \\
\text { Concordance (YTC) }\end{array}$ & IPC & $\begin{array}{l}\text { Canadian Standardized } \\
\text { Industrial Classification }\end{array}$ \\
\hline Johnson & 2002 & OECD Concordance & IPC & ISIC/SIC \\
\hline $\begin{array}{l}\text { Schmoch, Laville, } \\
\text { Patel e Frietsch }\end{array}$ & 2003 & DG Concordance & IPC & ISIC \\
\hline Lybbert e Zolas & 2014 & $\begin{array}{l}\text { Data Mining } \\
\text { Approach/Indexing and } \\
\text { Probabilistic Matching } \\
\text { Approach }\end{array}$ & IPC & $\begin{array}{l}\text { Aplicada às diversas } \\
\text { classificações } \\
\text { econômicas }\end{array}$ \\
\hline
\end{tabular}

Fonte: elaboração própria a partir de Lybbert e Zolas (2014) e Schmoch et al. (2003).

Com exceção da correspondência criada por Lybbert e Zolas (2014), todas as demais listadas na Tabela 1 sofrem de uma ou mais das limitações expostas a seguir (Lybbert \& Zolas, 2014, Schmoch, Laville, Patel, \& Frietsch, 2003). A primeira é a impossibilidade de terem aplicação internacional por serem atreladas a uma nação específica, na medida em que foram construídas com base em uma classificação das atividades econômicas utilizada em um único país. Isso é percebido ao observar-se, por exemplo, que Kortum e Putnam (1997) construíram uma concordância (chamada Yale Technology Concordance) baseada na associação entre a IPC e a Canadian Standard Industrial Classification (CSIC) que é aplicada apenas neste país. 
Tanto a Merit Concordance (Verspagen, Morgastel, \& Slabbers, 1994) quanto a DG Concordance (Schmoch et al., 2003) poderiam, em tese, ser replicadas em diferentes nações por basearem-se na International Standard Industrial Classification of All Economic Activities (ISIC) e na IPC, ambas adotadas por muitos países. Todavia, o problema é que as relações IPC/ISIC foram feitas manualmente por especialistas. Estes ligaram cada patente a uma atividade econômica, ou seja, uma a uma esta foi designada àquela (Schmoch et al., 2003). Nestes termos, há uma segunda limitação, já que a correspondência tem validade restrita ao período para o qual foi construída. Isso porque as dinâmicas tecnológica e econômica modificam-se ao longo do tempo, inclusive alterando o setor produtivo absorvedor de uma patente (Lybbert \& Zolas, 2014).

A terceira limitação pode ser percebida na correspondência de Johnson (2002), que partiu da Yale Technology Concordance (Kortum \& Putnam, 1997), cuja classificação econômica é a CSIC. Esta, então, foi conectada à ISIC, ou seja, a IPC foi relacionada à CSIC, que por sua vez foi conectada à ISIC. Nesse processo, a relação final (IPC-ISIC) perde acuidade e exatidão (Lybbert \& Zolas, 2014), tendo em vista que a ligação entre diferentes atividades econômicas é indesejável, pois cada uma delas representa uma estrutura econômica particular e, portanto, diferente entre si. Por fim, a quarta limitação refere-se à realização de correspondências em um nível de agregação amplo da classificação econômica. Isso impede uma compreensão mais detalhada entre a relação das tecnologias e das atividades econômicas (Schmoch et al., 2003).

Dadas as limitações mencionadas, restavam apenas duas alternativas para a realização de uma associação com um recorte específico (por exemplo, das patentes de uma universidade com as atividades econômicas de um país). A primeira seria adaptar uma correspondência já existente, como a YTC, assumindo sua defasagem temporal e a dificuldade de utilizá-la fora do contexto territorial em que fora criada. Ainda, ter-se-ia que lidar com a dificuldade de se ajustar duas classificações econômicas diferentes, cada uma delas referente a um país (Lybbert \& Zolas, 2014). A segunda alternativa, observar patente por patente e manualmente realizar a associação entre a classificação tecnológica e a econômica.

Todos os problemas acima relatados foram superados na correspondência proposta por Lybbert e Zolas (2014). A harmonização das patentes com o campo econômico construída por estes autores foi baseada em um método cuja aplicação 
pode se dar em diferentes contextos temporais e espaciais, diferentemente das anteriores. O método de Lybbert e Zolas (2014) está embasado em análises de textos, mineração de dados, matching probabilístico e programas computacionais, deixando a operação manual em escala mínima. Tal metodologia permite analisar a relação entre patentes e diversas atividades econômicas organizadas em diferentes tipos de classificação, tais como SITC, NACE, ISIC e HS ${ }^{5}$. Por essa razão ela pode ser aplicada amplamente e de forma direta em diversas situações, locais e períodos. Por essas vantagens tal método foi utilizado para analisar a relação entre a IPCUFRGS e a Divisão-CNAE.

\subsection{0 modelo "algorithimic links with probabilities"}

Lybbert e Zolas (2014) desenvolveram um modelo, genericamente denominado de "Algorithmic Links with Probabilities", que permite realizar a correspondência entre a International Standard Industrial Classification of All Economic Activities (ISIC) e a International Patent Classification (IPC). A ISIC ordena, de forma coerente e consistente, todas as atividades econômicas conforme regras, princípios e concepções acordadas internacionalmente. Sua estrutura é composta por quatro níveis de categorias hierárquicas. Ela começa pela "Seção" que organiza a produção em amplos clusters e é identificada por uma letra do alfabeto. Logo abaixo, posiciona-se a "Divisão", com um maior detalhamento. Posteriormente, o "Grupo" esmiúça ainda mais o sistema produtivo. Por fim, as "Classes" pormenorizam a classificação de todos os produtos e serviços da economia (UNITED NATIONS, 2008). Toda essa estruturação está exemplificada na Tabela 2, cuja linha do topo é um código referente às bebidas destiladas.

5 O significado de cada sigla: Standard International Trade Classification (SITC), European Classification of Economic Activities (NACE), International Standard Industrial Classification of all Economic Activities of the United Nations (ISIC) e Harmonized Commodity Description and Coding System of the World Customs Organization (HS). Todavia, dado seu objetivo, o artigo referencia apenas a ISIC. 


\section{Tabela 2}

Exemplo da estrutura da ISIC aplicada a uma atividade econômica ISIC - exemplo: C11.11-9

\begin{tabular}{c|c|c}
\hline Seção & C & Indústria de transformação \\
\hline Divisão & 11 & Fabricação de bebidas \\
\hline Grupo & 1 & Fabricação de bebidas \\
\hline Classe & $1-9$ & Fabricação de destilados \\
\hline
\end{tabular}

Fonte: elaboração própria a partir da ISIC (2008).

Vários países aceitam a ISIC como um padrão para categorizar suas atividades de produção. Esse é o caso do Brasil, onde a Classificação das Atividades Econômicas (CNAE) 2.0 é baseada na ISIC 4.0. O primeiro e o segundo nível daquela, respectivamente - Seção e Divisão - são idênticos aos desta. Nos demais dígitos (Grupo e Classe), há peculiaridades inerentes ao sistema produtivo brasileiro, mas mesmo neste caso há pouca diferença com relação à ISIC (UNITED NATIONS, 2016).

Já a International Patent Classification (IPC) foi adotada em 1971 pelo acordo de Strasbourg. Ela é uma linguagem de símbolos (numéricos e de letras) que formam um sistema hierárquico de áreas tecnológicas utilizado para a classificação de patentes e modelos de utilidades. Para exemplificar, a Tabela 3 apresenta a classificação "H 03 F 3/187", referente às tecnologias de circuitos integrados. Neste caso, o H é a "Seção", 03 a "Classe", F a "Subclasse" e 3/187 o "Grupo".

\section{Tabela 3}

Exemplo: estrutura hierárquica da Internacional Patent Classification H 03 F 3/187

\begin{tabular}{c|c}
\hline Seção $\mathrm{H}$ & Eletricidade \\
\hline Classe 03 & Circuitos eletrônicos básicos \\
\hline Subclasse $\mathrm{F}$ & Amplificadores \\
\hline Subgrupo $3 / 187$ & em circuitos integrados \\
\hline
\end{tabular}

Fonte: elaboração própria a partir da IPC 2016.

Lybbert e Zolas (2014) propuseram duas maneiras para realizar a conexão entre tecnologia e áreas econômicas. A primeira ligou a ISIC à IPC, enquanto a segunda faz o caminho contrário, associando a IPC à ISIC. A construção de ambas ocorre com a mesma lógica e é apresentada a seguir. 


\subsubsection{Correspondência da IPC à ISIC}

Cada código da IPC pode estar associado a diversas atividades econômicas da ISIC, de acordo com diferentes probabilidades. Lybbert e Zolas (2014) iniciaram o cálculo desta probabilidade através da técnica de mineração ${ }^{6}$. Eles observaram as descrições de cada atividade econômica e, então, extraíram termos de busca (palavras-chaves) que as representam. Depois, voltaram-se para os títulos e resumos das patentes, buscando neles as expressões representativas (palavraschaves) das atividades econômicas. Por fim, foi feita a frequência ponderada das associações encontradas da seguinte forma:

$$
\text { Frequência ponderada específica }\left(\operatorname{SITC}_{i}, I P C_{j}\right)=\frac{m_{i j} / N_{j}}{M_{i}}
$$

onde $m_{i j}$ indica o número de patentes que contêm a subclasse (da IPC) $j$ dentre todas as que afloraram ao utilizar-se a palavra-chave referente à atividade econômica $i$ da ISIC; $N_{j}$ representa o número total de patentes da base PATENTSCOPE $^{7}$ que contêm a subclasse $j ; M_{i}$ é o número total de patentes, de diferentes classificações, que irrompem com o termo de busca representativo da categoria $i$ da ISIC. Ressalva-se que existem, em muitos casos, múltiplos códigos de IPC associados a uma única patente, assim, cada um deles foi igualmente ponderado, de acordo com a equação (1). Para completar, as frequências específicas foram normalizadas, permitindo que suas somas resultem em 1.

Todavia, o apresentado esquema de pesos (1) sobrevaloriza as subclasses das IPC pouco empregadas frente àquelas frequentemente utilizadas, o que prejudica a verdadeira importância das diferentes tecnologias classificadas via IPC. Assim, para evitar tal problema, uma ponderação híbrida alternativa foi aplicada à frequência, conforme fórmula a seguir:

\footnotetext{
${ }^{6}$ Mineração de dados é um método aplicado sob controle humano, para organizar informações de forma manual e resulta em algoritmos que permitem tanto analisar dados, como a partir deles construir padrões em categorias específicas (Amatriain \& Pujol, 2015).

${ }^{7} \mathrm{O}$ banco de dados PATENTSCOPE está disponível em: <http://www.wipo.int/patentscope/en/>.
} 


$$
\text { Frequência ponderada híbrida }\left(\operatorname{SITC}_{i}, I P C_{i j}\right)=\frac{s_{i j} m_{i j}}{\sum_{j} s_{i j} m_{i j}}
$$

onde $s_{i j}$ representa a participação das patentes na subclasse $j$ cuja atividade econômica ordenada é a $i$. Essa última forma de calcular a frequência ponderada (2), ao considerar o peso de cada patente na sua respectiva subclasse $j$, reflete melhor a importância relativa de cada tecnologia no Sistema de Inovação e, assim, torna mais acurado o processo de correspondência entre patentes e indústria.

No desenvolvimento do referido algoritmo, os autores não limitaram as patentes por um determinado ano ou país e, assim, trabalharam com um volume grande e variado de dados. As patentes pesquisadas vieram da base da World Intellectual Property Organization (WIPO), a PATENTSCOPE. Já as descrições das atividades econômicas foram retiradas da ISIC.

\subsubsection{Correspondência da ISIC à IPC}

A Harmonização da ISIC com a IPC através de uma metodologia que parte desta para aquela segue a mesma lógica já descrita. A diferença é que neste caso extraiu-se da IPC, inicialmente, algumas expressões que as definissem em palavraschaves. Estas, posteriormente, foram procuradas nas descrições de cada atividade econômica da ISIC. Com os encontros de palavras, fruto do cruzamento entre ambas classificações, foi feita a frequência ponderada seguindo a mesma lógica utilizada nas equações (1) e (2), apresentadas anteriormente.

Cada IPC é associada a uma classificação ISIC com diferente probabilidade, respeitando-se a participação individual da patente com relação ao total de patentes do país ou organização. Desta forma, a maior presença de determinada IPC amplia a sua probabilidade relativa de associação com certa atividade econômica; consequentemente, a tecnologia representada por tal IPC ganha maior peso. Isso amplia o destaque dos campos de pesquisa e ciência que mais invenções realizam. A Tabela 4 apresenta um exemplo do resultado final da correspondência desenvolvida Lybbert e Zolas $(2014)^{8}$.

\footnotetext{
${ }^{8}$ A correspondência probabilística completa entre a IPC e a ISIC construída por Lybbert e Zolas (2014) pode ser visualizada no sítio da WIPO (2016) disponível em:<http://www.wipo.int/publications/en/details.jsp?id=3949\&plang=EN>.
} 


\section{Tabela 4}

Exemplo da correspondência probabilística

\begin{tabular}{c|c|c}
\hline Código IPC - nível subclasse & Código ISIC - nível classe & Probabilidade \% \\
\hline A01B & 0150 & 20,3 \\
\hline A01B & 0161 & 9,9 \\
\hline A01B & 0164 & 30,4 \\
\hline A01B & 0210 & 3,3 \\
\hline A01B & 2821 & 30,5 \\
\hline A01B & 2822 & 5,4
\end{tabular}

Nota. Fonte: elaboração própria a partir de Lybbert e Zolas (2014).

O código IPC A01B, primeira coluna da Tabela 4, é ligado a diferentes classificações da ISIC, apresentadas na segunda coluna, de acordo com as probabilidades da terceira coluna. Isso significa que as atividades econômicas associadas com pesos maiores têm uma associação mais forte com as tecnologias representadas pelas IPC A01B.

A metodologia ora apresentada pode ser aplicada no contexto de uma única organização, inclusive pelas universidades. Estas podem identificar as possíveis atividades econômicas que têm possibilidade técnica de absorverem suas tecnologias. Isso daria suporte aos estudos de inteligência competitiva na área de gestão de negócios tecnológicos que visem "desengavetar" as patentes. A exploração de tal ferramenta para as patentes da UFGRS é experimentada na próxima subseção.

\section{Metodologia}

O principal objetivo do artigo é realizar a correspondência entre os códigosIPC $^{9}$ das patentes da UFRGS com as atividades econômicas da CNAE. Para tanto, foi realizado um levantamento das patentes e definido o nível hierárquico da CNAE que será utilizado para o cruzamento com a IPC.

As patentes da UFRGS com seus respectivos códigos IPC foram extraídas do Orbit, sistema de banco de dados comercial que gera informações on-line sobre ativos intelectuais protegidos, no mês de maio de 2016. Não houve limitação de período para se trabalhar com o conjunto integral de patentes depositadas e

\footnotetext{
${ }^{9}$ Neste artigo, foi usado a IPC vigente no primeiro semestre de 2016, a qual continha 8 Seções, 129 Classes e 640 Subclasses, além de milhares de subgrupos (uma divisão específica dentro das subclasses).
} 
publicadas até a citada data, um total de $344^{10}$. Em seguida, aplicou-se a correspondência probabilística criada por Lybbert e Zolas (2014) para relacionar as IPCs extraídas das patentes da UFRGS à classificação CNAE, respeitando-se os pesos da participação de cada código da IPC em diversas divisões da CNAE ${ }^{11}$. Esta, como já informado, é idêntica à ISIC no primeiro e no segundo nível. Por isso, a correspondência daqueles autores referente à IPC com a ISIC pode ser, ipsis litteris, aplicada àquela entre IPC e a CNAE, nos dois referidos níveis.

A associação entre os símbolos da IPC das patentes da UFRGS e as Divisões da CNAE foi representada em uma tabela, arranjando-se as indústrias nas colunas e os códigos tecnológicos nas linhas. Com o intuito de explorar a referida associação, foi feita uma análise multivariada, por meio de uma Análise de Correspondência (AC), e um teste Qui Quadrado. O software estatístico utilizado foi o SPSS.

A técnica de $A C$ foi utilizada como forma de melhorar a visualização e a extração de informações dos dados. Ela auxiliou na verificação das áreas em que a UFRGS mantém produção tecnológica protegida e das potenciais indústrias de aplicação dessas invenções. A AC é útil para interpretar dados de tabela de contingência e, a partir deles, gerar hipóteses exploratórias, verificando possíveis associações entre as variáveis categóricas envolvidas e a relação entre linhas e colunas (Aguayo, 1993). Tais padrões de vinculação podem ser testados com experimentos confirmatórios posteriores, empregando-se as técnicas usuais da estatística.

Uma tabela de contingência com um grande número de linhas e colunas é de complexa representação, tendo em vista o enorme volume de pontos dispersos em diferentes espaços geométricos, o que torna complicada a interpretação dos dados. Para superar essa dificuldade, técnicas como a AC reduzem a dimensionalidade desta nuvem de pontos a um subespaço ótimo de menor dimensão (Padro, 2012).

\footnotetext{
${ }^{10}$ É uma limitação desse trabalho o fato de que o Orbit extrai apenas um único símbolo IPC por patente, apesar de o INPI designar, geralmente, mais de um para cada patente. Com isso, a correspondência entre IPC/UFRGS e CNAE, efetivada neste artigo, considera apenas um único código IPC por patente. Porém, na maioria das vezes os diferentes códigos IPC associados a cada patente são idênticos no nível de Seção, Classe e Subclasse, modificando-se com mais frequência nos Subgrupos. Por isso, os resultados da correspondência apresentados aqui não iriam modificar-se significativamente, mesmo que se considerasse todas as IPCs utilizadas para classificar cada patente da UFRGS.

${ }^{11}$ Seja com as patentes da UFRGS, seja com as patentes usadas por Lybbert e Zolas (2014), em ambos os casos a correspondência encontrará as mesmas atividades econômicas. Todavia, os pesos na distribuição do código da IPC entre diversas atividades econômicas tenderiam a ser diferentes, conforme a base de dados das patentes.
} 
Nesse trabalho a $A C$ foi usada para construir um mapa da vinculação entre os códigos da IPC das patentes da UFRGS e as Divisões-CNAE da Indústria. Isso porque essa representação gráfica aproxima geometricamente, no espaço, a posição relativa das variáveis categóricas em estudo (Prado, 2012). Portanto, utilizou-se uma representação em figura que agrupou as patentes e as atividades econômicas vinculadas entre si.

Posteriormente, um teste Qui Quadrado foi aplicado à tabela de contingência que apresenta a relação entre a IPC das patentes da UFRGS com as Divisões da CNAE. Isso foi feito porque essa técnica estatística permite analisar se há um padrão de associatividade entre dados categóricos distintos, ou seja, se eles são independentes ou não. Assim, utilizando-se desse instrumento, foi possível avaliar, estatisticamente, a existência de uma relação entre a IPC e a CNAE.

Através do teste Qui Quadrado foi testada a expectativa de que as invenções tecnológicas produzidas pela UFRGS seriam aplicadas com a mesma probabilidade em qualquer indústria da CNAE. Neste caso, poder-se-ia esperar que cada DivisãoCNAE fosse igualmente potencial receptora de qualquer patente da UFRGS. Em outras palavras, formulou-se a seguinte pergunta: as criações patenteadas pela UFRGS são associadas a qualquer tipo de indústria ou apenas a algumas específicas?

O primeiro passo para responder a essa indagação é calcular a tabela do valor esperado correspondente à tabela original que associa os dados das IPC-UFRGS com os da Divisão-CNAE. O valor esperado de cada célula pertinente é calculado como $\frac{L i * C j}{N}$, onde $L i$ representa a soma da linha $i ; C$ a soma da coluna $j$ e $N$ é o total considerado do número de patentes da UFRGS. O teste Qui Quadrado é definido como $x^{2}=\sum \sum \frac{\left(o_{i j}-E_{i j}\right)^{2}}{E_{i j}}$, onde $O$ é o valor observado referente a linha $i$ e coluna $j$; e $E$ é o valor esperado da linha $i$ e coluna $j \quad$ (Howell, 2011). O resultado advindo do cálculo do Qui Quadrado será o valor da estatística do teste que será comparado com o valor crítico (retirado da tabela de distribuição padrão), considerando-se 5\% de nível de significância, com (c-1).(l-1) graus de liberdade, sendo $c$ o número de colunas e $I$ o número de linhas da tabela de contingência. Isso possibilitará a rejeição (ou não) da hipótese nula de que não existe uma associação entre as IPC- 
UFRGS e as diversas Divisões-CNAE. Automaticamente, a hipótese alternativa é de que há uma associação entre as IPC-UFRGS e as diversas Divisões-CNAE.

Com as ferramentas estatísticas apresentadas, busca-se explorar metodologias úteis à visualização da aplicação das patentes universitárias junto às atividades econômicas. Isso poderá ser um instrumento para que a gestão tecnológica concentre-se em setores econômicos mais aptos a desenvolverem negócios e, assim, "desengavete" suas patentes em processos eficientes de negócios.

\section{Análise e Discussão dos Resultados}

O resultado da aplicação da correspondência probabilística criada por Lybbert e Zolas (2014) está na Tabela 5. Nesta tabela a primeira linha registra os códigos das atividades econômicas no nível da Divisão-CNAE e a primeira coluna os da IPC das patentes da UFRGS ${ }^{12}$. Cada célula contém o número de patentes cujo campo tecnológico está associado a determinada área produtiva ${ }^{13}$.

O primeiro destaque é que $45 \%$ das patentes da UFGRS estão associadas a apenas duas atividades econômicas, "fabricação de produtos químicos" (Divisão 20) e "fabricação de produtos farmo-químicos e farmacêuticos" (Divisão 21). A primeira acopla 83 e a segunda 72 patentes (números visualizados na última linha da Tabela 5). Em terceiro lugar, com 46 patentes (13\% do total), está "agricultura, pecuária e serviços relacionados" (Divisão 01). Posteriormente, "extração de petróleo e gás natural" (Divisão 6), "fabricação de produtos de metal, exceto máquinas e equipamentos" (Divisão 25) e "fabricação de máquinas e equipamentos" (Divisão 28) contêm, cada uma delas, 20 patentes, o que, individualmente, corresponde a $6 \%$ do total.

Evidencia-se que $76 \%$ das patentes da UFRGS estariam conjugadas a apenas 6 das 87 Divisões da CNAE 2.0. Isso sinaliza que o foco da estratégia de transferência tecnológica da UFRGS seria um rol de atividades econômicas

12 O anexo I descreve cada uma das atividades econômicas, no nível de Divisão-CNAE, representadas pelos números da primeira linha da Tabela 5; da mesma forma, o anexo II o faz para cada símbolo de patente alocado na primeira coluna.

${ }^{13}$ Nos casos em que a ponderação resultou em um total de patentes inferior a 0,5 , foi atribuído o valor zero para a célula. Aos valores acima de 0,5 e abaixo de 1 foi atribuído o valor 1 . 
específicas, principalmente aquelas voltadas para bens e produtos ligados à química, agropecuária, minério e máquinas e equipamentos.

O segundo destaque é que $22 \%$ das patentes da UFRGS estão alocadas em apenas duas Subclasses-IPC, "Preparações para finalidades médicas, odontológicas ou higiênicas" (A61K) e "atividade terapêutica específica de compostos químicos ou preparações medicinais" (A61P). Aquela acopla 45 e esta 33 patentes (números visualizados na última coluna da Tabela 5). Em terceiro lugar, com 23 patentes (7\% do total) está "micro-organismos ou enzimas; suas composições" (C12N). Posteriormente, "investigação ou análise dos materiais pela determinação de suas propriedades químicas ou físicas" (G01N) registra 21 patentes (6\% do total), seguida dos "processos químicos ou físicos, p. ex. catálise, química coloidal; aparelhos pertinentes aos mesmos" (B01J), onde alocam-se 19 patentes (5\% do total).

Sobressai o fato de que $40 \%$ das patentes da UFRGS estão classificadas em apenas 5 das 640 Subclasses da IPC, o que sinaliza que as patentes desta universidade estão focadas em áreas específicas e/ou especializadas. Complementarmente, a ciência química está concatenada com as cinco Subclasses principais, citadas no parágrafo anterior, nas quais a UFRGS produz mais patentes. Isso corrobora o resultado anterior sobre os campos econômicos cujas correspondências com as patentes-UFRGS seriam as maiores: "fabricação de produtos químicos" e "fabricação de produtos farmo-químicos e farmacêuticos". 


\section{Tabela 2}

Correspondência entre a IPC das patentes da UFRGS e a Divisão/CNAE

\begin{tabular}{|c|c|c|c|c|c|c|c|c|c|c|c|c|c|c|c|c|c|c|c|c|c|c|c|c|}
\hline & 01 & 02 & 06 & 08 & 10 & 11 & 13 & 18 & 19 & 20 & 21 & 22 & 23 & 24 & 25 & 26 & 27 & 28 & 32 & 35 & 36 & 37 & 43 & \begin{tabular}{|l} 
soma \\
linha \\
\end{tabular} \\
\hline $01 \mathrm{H}$ & 5 & 2 & 0 & 0 & 0 & 0 & 0 & 0 & 0 & 0 & 0 & 0 & 0 & 0 & 0 & 0 & 0 & 0 & 0 & 0 & 0 & 0 & 0 & \\
\hline $\mathrm{A} 01 \mathrm{~N}$ & 2 & 0 & 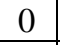 & $\underline{0}$ & 0 & 0 & 0 & 0 & 0 & 7 & 0 & 0 & 0 & 0 & 0 & 0 & 0 & 0 & $\rho_{-}$ & 0 & 0 & 0 & 0 & 0 \\
\hline $\mathrm{A} 01 \mathrm{P}$ & 2 & 0 & 0 & 更 & 0 & 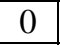 & 0 & 0 & 0 & 3 & 0 & 40 & 4 & 4 & 0 & 0 & 0 & 0 & 0 & 0 & 0 & 0 & 0 & 6 \\
\hline A61K & 12 & 0 & 0 & 0 & 2 & 0 & 0 & 0 & 0 & 0 & 31 & 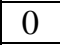 & 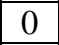 & 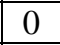 & 0 & 0 & 0 & 0 & 0 & 0 & 0 & 0 & 0 & 45 \\
\hline A61L & 1 & 0 & 0 & 0 & 0 & , & 1 & 0 & 0 & 2 & 0 & 0 & 0 & 0 & 0 & 0 & 0 & 0 & 1 & 0 & 0 & 0 & 1 & 7 \\
\hline A61P & 11 & 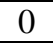 & 0 & 0 & 8 & 0 & 0 & 0 & 0 & 0 & 20 & 0 & 0 & 0 & 0 & 0 & 0 & 0 & 0 & 0 & 0 & 0 & 0 & 33 \\
\hline B $01 \mathrm{~J}$ & 0 & 0 & 8 & ( & 0 & 0 & 0 & 0 & 1 & 6 & 0 & 0 & 0 & 0 & 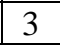 & 0 & 0 & 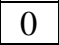 & 0 & 1 & 0 & 0 & 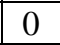 & 19 \\
\hline B29C & 0 & 0 & 0 & 0 & 0 & 0 & 1 & 0 & 0 & 1 & 0 & 2 & 0 & 0 & 0 & 0 & 0 & 2 & 0 & 0 & 0 & 0 & 0 & 6 \\
\hline $\mathrm{B} 82 \mathrm{~B}$ & 0 & 0 & 0 & 0 & 0 & 0 & 0 & 0 & 0 & 3 & 0 & 0 & 0 & 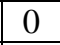 & 3 & 1 & 0 & 1 & 0 & 0 & 0 & 0 & 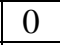 & 8 \\
\hline $\mathrm{B} 82 \mathrm{Y}$ & 0 & 0 & 0 & 0 & 0 & 0 & 0 & 0 & 0 & 0 & 0 & 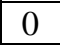 & 4 & 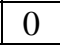 & 2 & 1 & 0 & 0 & 3 & 0 & 0 & 0 & 0 & 8 \\
\hline $\mathrm{C} 01 \mathrm{~B}$ & 0 & 0 & 3 & 0 & 0 & 0 & 0 & 0 & 0 & 2 & 0 & 0 & 0 & 0 & 0 & 0 & 0 & 0 & 0 & 1 & 0 & 0 & 0 & 7 \\
\hline $\mathrm{C} 02 \mathrm{~F}$ & 0 & 0 & 0 & 0 & 0 & \begin{tabular}{|l|l|} 
\\
\end{tabular} & 0 & 0 & 0 & 0 & 0 & 0 & 0 & 0 & 0 & 0 & 0 & 0 & 0 & 0 & 1 & 9 & 0 & 10 \\
\hline $\mathrm{C} 07 \mathrm{C}$ & 0 & 0 & 3 & 1 & 0 & 1 & 0 & 0 & 3 & 8 & 0 & 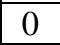 & 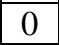 & 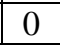 & 0 & 0 & 0 & 0 & 0 & 0 & 0 & 0 & 0 & 16 \\
\hline C07D & 4 & 0 & 0 & 0 & 0 & 0 & 0 & 0 & 0 & 3 & 7 & 0 & 0 & 0 & 0 & 0 & 0 & 0 & 0 & 0 & 0 & 0 & 0 & 14 \\
\hline $\mathrm{C} 07 \mathrm{~F}$ & 0 & 0 & 0 & 1 & 0 & 0 & 0 & 0 & 0 & 4 & 0 & 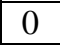 & 0 & 0 & 0 & 0 & 0 & 0 & 0 & 0 & 0 & 0 & 0 & 5 \\
\hline $\mathrm{C} 07 \mathrm{~K}$ & 1 & 0 & 0 & 0 & 0 & 0 & 0 & 0 & 0 & 0 & 6 & 0 & 更 & 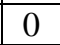 & 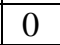 & 4 & 0 & 0 & 0 & 0 & 0 & 0 & 0 & 8 \\
\hline $\mathrm{C} 08 \mathrm{~F}$ & 0 & 0 & 0 & 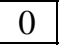 & 0 & 0 & 0 & 0 & 0 & 11 & 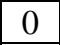 & 0 & 0 & 0 & 0 & 0 & 0 & 0 & 0 & 0 & 0 & 0 & 0 & 11 \\
\hline $\mathrm{C} 08 \mathrm{G}$ & 0 & 0 & 0 & 0 & 0 & 0 & 0 & 0 & 2 & 3 & 0 & 0 & 0 & 0 & 0 & 0 & 0 & 0 & 0 & 0 & 0 & 0 & 0 & 6 \\
\hline C08J & 0 & 0 & 0 & 0 & 0 & 0 & 0 & 0 & 1 & 5 & 0 & 1 & 0 & 0 & 0 & 0 & 0 & & 0 & D & 0 & 0 & 0 & 8 \\
\hline $\mathrm{C} 08 \mathrm{~K}$ & $\Omega$ & $\Omega$ & 0 & 1 & 0 & 0 & 0 & 0 & 1 & 8 & 0 & 2 & 0 & 0 & 0 & 0 & 0 & 0 & 0 & 0 & 0 & 0 & 0 & 12 \\
\hline $\mathrm{C} 08 \mathrm{I}$ & 0 & 0 & 0 & 0 & 0 & 0 & 0 & 0 & 0 & 7 & 0 & 2 & 0 & 0 & 0 & 0 & 0 & 0 & 0 & 0 & 0 & 0 & 0 & 10 \\
\hline $\mathrm{C} 09 \mathrm{D}$ & 0 & 0 & 0 & 0 & 0 & 0 & 0 & 0 & 0 & 4 & 0 & 0 & 0 & 0 & 1 & 0 & 0 & 0 & 0 & 0 & 0 & 0 & 0 & 5 \\
\hline $\mathrm{C} 12 \mathrm{~N}$ & 8 & 0 & 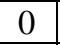 & 4 & 7 & 0 & 0 & 0 & 0 & 2 & 0 & 0 & 0 & 10 & 0 & 0 & 0 & 0 & 0 & 0 & 0 & 0 & 0 & 23 \\
\hline $\mathrm{C} 12 \mathrm{Q}$ & 0 & 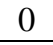 & 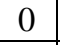 & 0 & 0 & 0 & 0 & 0 & 0 & 0 & 0 & 0 & 0 & 0 & 0 & 0 & 0 & 5 & 0 & 0 & 0 & 0 & 0 & 7 \\
\hline $\mathrm{C} 23 \mathrm{C}$ & 0 & 0 & 0 & 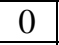 & 0 & 0 & 0 & 0 & 0 & 0 & 0 & 0 & 0 & 2 & 4 & 0 & 0 & 0 & 0 & 0 & 0 & 0 & 0 & 7 \\
\hline $\mathrm{C} 25$ & 0 & 0 & 0 & 0 & 0 & $\begin{array}{ll}0 \\
\end{array}$ & 0 & 0 & 0 & 2 & 0 & 0 & 0 & 2 & 1 & 0 & 0 & \begin{tabular}{|l|}
0 \\
\end{tabular} & 0 & 0 & 0 & 0 & 0 & 6 \\
\hline $\mathrm{C} 25$ & 0 & 0 & 0 & 0 & 0 & 0 & 0 & 0 & \begin{tabular}{|l|}
0 \\
\end{tabular} & 0 & 0 & 0 & 0 & 2 & 5 & \begin{tabular}{|l|}
0 \\
\end{tabular} & 0 & 0 & 0 & 0 & 0 & 0 & 0 & 8 \\
\hline G01F & 0 & 0 & 0 & 0 & 0 & 0 & 0 & 0 & 0 & 0 & 0 & 0 & 0 & 0 & 0 & 3 & 0 & 2 & 0 & 0 & 0 & 0 & 0 & 5 \\
\hline 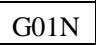 & 0 & 0 & 5 & 0 & 0 & 0 & 0 & 0 & 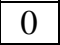 & 4 & 0 & 0 & 0 & 1 & 0 & 5 & 0 & 10 & 0 & 0 & 0 & 0 & 0 & 21 \\
\hline & 0 & 0 & 0 & 0 & 0 & 0 & 0 & 0 & 0 & 0 & \begin{tabular}{|l|}
0 \\
\end{tabular} & 0 & \begin{tabular}{|l|}
0 \\
\end{tabular} & 0 & 0 & 0 & 6 & 0 & 0 & 0 & 0 & 0 & 0 & 7 \\
\hline luna & 46 & $T$ & 20 & $J$ & 10 & 1 & 2 & 0 & 9 & 83 & 72 & 7 & 3 & 7 & 20 & 10 & 7 & 20 & 4 & 2 & 1 & 9 & 1 & 344 \\
\hline
\end{tabular}

Nota. Fonte: Dados da pesquisa.

O fato de que as atividades inventivas da UFRGS ocorrem em áreas específicas e especializadas em certos campos científicos também pode ser constatado ao observar-se que as patentes estão alocadas em apenas 15 das 129 Classes da IPC, (11,6\% do total das Classes). Em um nível maior de desagregação, existem patentes dessa instituição em apenas 30 das 640 Subclasses $(4,6 \%$ do total das Subclasses).

A Figura 1 apresenta a Análise de Correspondência feita a partir da Tabela $5^{14}$. As maiores aproximações relativas entre os símbolos IPC-UFRGS e as Divisões-

\footnotetext{
${ }^{14}$ Para melhor visualização no gráfico de análise de correspondência, foram excluídas as patentes referentes às classificações C02F (tratamento de água, de águas residuais, de esgotos ou de lamas e
} 
CNAE representam a associação destas duas estruturas. A AC ratifica o resultado de que a ciência química responde por grande parte das patentes da UFRGS, sendo os ramos econômicos "fabricação de produtos químicos" e "fabricação de produtos farmo-químicos e farmacêuticos" os principais absorvedores das patentes desta universidade. As cinco maiores correspondências em termos de números de patentes são destacadas na figura e serão detalhadas a seguir.

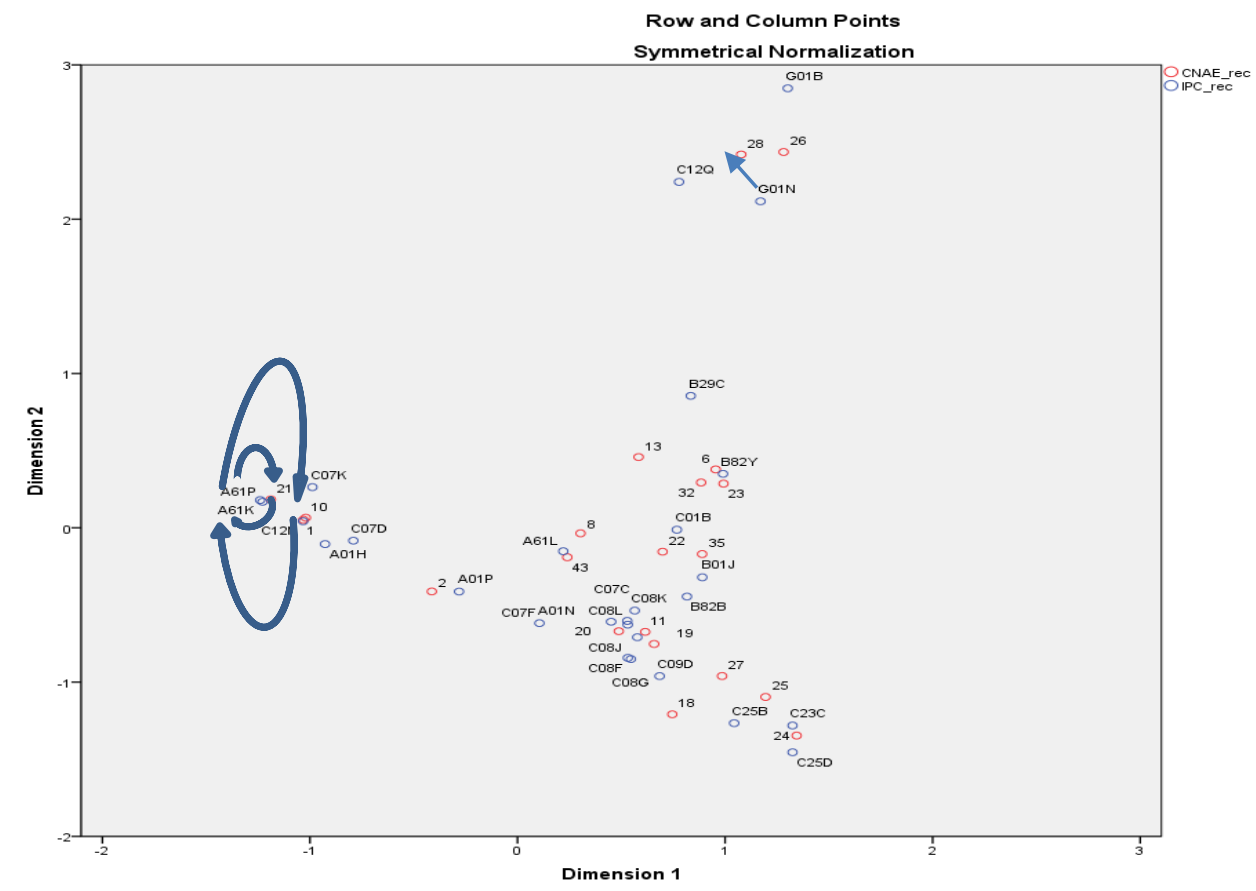

Figura 1

Análise de correspondência: patentes da UFRGS e as Divisões/CNAE Fonte: dados da pesquisa com geração automatizada no programa SPSS.

A maior correspondência observada ocorre entre "Preparações para finalidades médicas, odontológicas ou higiênicas" (IPC-A61K) e "Fabricação de produtos farmoquímicos e farmacêuticos" (Divisão-CNAE 21), com 31 patentes. Já a segunda maior é entre "atividade terapêutica específica de compostos químicos ou preparações medicinais" (IPC-A61P) e novamente "fabricação de produtos farmoquímicos e farmacêuticos" (Divisão-CNAE 21), com 20 patentes. Isso evidencia um significativo potencial de criação de produtos químicos voltados à área médica, ou seja, produção de medicamentos.

lodos) e H01M (processos ou meios, p. ex. baterias, para a conversão direta da energia química em energia elétrica). 
Na sequência, a associação entre "Preparações para finalidades médicas, odontológicas ou higiênicas" (IPC-A61K) liga-se à classe econômica "agricultura, pecuária e serviços relacionados" (Divisão-CNAE 01), com 12 patentes. Sucessivamente, "atividade terapêutica específica de compostos químicos ou preparações medicinais" (IPC-A61P) está ligada à "agricultura, pecuária e serviços relacionados" (Divisão-CNAE 01), com 11 patentes. Isso sugere que a terceira e quarta maiores ligações têm um viés para produtos de defensivos agrícolas e vacinas veterinárias.

O quinto vínculo refere-se àquele entre "investigação ou análise dos materiais pela determinação de suas propriedades químicas ou físicas" (IPC-G01N) e a atividade "fabricação de máquinas e equipamentos" (Divisão-CNAE 28). Isso pode ser uma revelação de um potencial para criação de máquinas, equipamentos ou instrumentos úteis para investigações das propriedades de diferentes materiais. Também nesse caso a química está presente, relacionando-se à engenharia de materiais.

A análise exploratória realizada acima propôs relações específicas entre as IPCUFRGS e a Divisão-CNAE que podem ser testadas por um teste Qui Quadrado. Tal ferramenta estatística permite responder à pergunta formulada anteriormente: as criações patenteadas pela UFRGS são associadas a qualquer tipo de indústria ou apenas a algumas específicas? Considerando-se a hipótese nula de que não existe uma associação entre as IPC-UFRGS e as diversas Divisões-CNAE, o resultado do teste estatístico apresentou $x^{2}=2.195,538$ com 638 graus de liberdade e probabilidade de significância (valor-p) bem abaixo de $5 \%$. Assim, pode-se rejeitar a hipótese nula de que não existe uma associação entre as IPC das patentes da UFRGS e as Divisões-CNAE.

Em outras palavras, existe uma relação significativa entre as patentes da UFRGS e as atividades econômicas.

\section{Considerações Finais}

Este artigo utilizou uma metodologia que possibilita a ligação técnica entre as patentes de uma universidade e as atividades econômicas. Assim, sugere aos Núcleos de Inovação Tecnológica (NITs) um critério para elaboração de estudos de inteligência competitiva voltados à transferência de tecnologia. Considera-se que a 
gestão tecnológica das ICT será mais eficaz na medida em que sejam identificados os setores produtivos com maior potencial de efetivamente avançarem em uma parceria e, assim, tornarem-se absorvedores das inovações das instituições de pesquisas. Desta forma, a ferramenta que permite visualizar campos de aplicação para todas as patentes é importante para o "desengavetamento" delas.

Uma vez que a ciência já é um insumo importante para as atividades econômicas, a transferência de conhecimento das universidades é um tema relevante. Por isso, cada vez mais os gestores das universidades preocupam-se com as possíveis aplicações econômicas das invenções que resultam das pesquisas. Neste caso, cabe aperfeiçoar os mecanismos de interação entre estas organizações.

O Brasil já encoraja, via políticas públicas, a comercialização das invenções das ICT, inclusive permitindo retornos financeiros advindos da pesquisa acadêmica. Em que pese a oposição a tal modelo, é fato que ele já avançou nos países desenvolvidos, com resultados positivos para a inovação. Inclusive, é recorrente a presença de alguma universidade, com intensa interação com empresas de base tecnológica, em territórios que contenham clusters de inovação. Mesmo que a negociação dos ativos intelectuais das universidades torne-se cada vez mais comum, a relação universidade-empresa carece de estudos que possam esclarecer como tal interação pode ser estabelecida de maneira a beneficiar a sociedade. No Brasil, os NTIs possuem pouca experiência no processo de transferência de tecnologia e clamam por mecanismos que possam impulsioná-lo. Disso decorre a importância de aprofundar o debate sobre ferramentas que possibilitem a conexão entre as criações oriundas das pesquisas universitárias e o sistema produtivo.

Esse trabalho resgatou a discussão internacional sobre correspondência da IPC com atividades econômicas, apresentou o estado da arte, e fez uma primeira experimentação com as patentes da UFRGS, como um passo inicial de conexão do conhecimento gerado pela universidade com a atividade econômica. Todavia, muito trabalho ainda pode ser feito, e isso inclui a construção de uma correspondência genuinamente brasileira, com base nos dados de patentes de todo o Brasil, e a realização de análises semelhantes para outras ICT.

A metodologia utilizada para realizar a correspondência entre as patentes da UFRGS e a CNAE está em um estágio inicial de discussão na WIPO, mas mostra-se 
relevante para aprimorar a transferência tecnológica ao construir um mapa contendo a associatividade entre tecnologias e indústrias específicas. Apesar dessa discussão ocorrer em âmbito internacional, o presente estudo é o primeiro que busca utilizar a referida correspondência nas patentes brasileiras.

O método de correspondência entre a IPC e as atividades econômicas, desenvolvido por Lybbert e Zolas (2014), foi o primeiro a automatizar tal processo, antes feito manualmente e segundo o julgamento do pesquisador. $O$ presente artigo procurou oferecer uma contribuição a essa agenda de pesquisa ao realizar uma aplicação desse método para um caso brasileiro, testando estatisticamente sua validade. Foi possível concluir que existe uma relação significativa entre as patentes da UFRGS e as atividades econômicas (indústria) específicas. Além disso, na análise de correspondência visualizou-se de maneira mais clara a relação entre patentes e atividade econômica. Como ponte com a realidade, sugere-se ao NIT da UFRGS desenvolver estudos de mercado e ações de interação com empresas incluídas nas atividades econômicas mapeadas por este artigo. Em síntese, os resultados apontaram que as tecnologias da UFRGS relacionadas às áreas médicas, odontológicas ou higiênicas e terapêuticas teriam aplicação nas indústrias química e de farmácia, em primeiro lugar, e nas atividades agropecuárias, secundariamente. Neste último caso, destaca-se o potencial para o desenvolvimento de defensivos agrícolas e vacinas animais. Complementarmente, as tecnologias de engenharia de material estão associadas às indústrias de máquinas e equipamentos.

\section{Referências}

Aguayo, M. T.V. (1993). Análise de Correspondência e Modelos Log-Lineares: um Enfoque Integrado para a Análise Exploratória de Dados Categóricos. (Tese de Doutorado, Universidade Estadual de Campinas, Campinas, SP, Brasil).

Amatriain, X., \& Pujol, J.M. (2015). Data mining methods for recommender systems. In Ricci, F., Rokach, L., Shapira, B. (Eds). Recommender systems handbook. Boston: Springer.

Apax Partners Ltd (2005). Understanding technology transfer. Recuperado em 19 setembro, 2017 , de http://www.wipo.int/export/sites/www/sme/en/newsletter/2011/attachments/apax_ tech_transfer.pdf 
Buenstorf, G., \& Geissler, M (2012). Not invented here: technology licensing, knowledge transfer and innovation based on public research. Journal of Evolutionary Economics, 22(3), 481-511.

Castro, B. S., \& Souza, G. C. (2012). O papel dos Núcleos de Inovação Tecnológica (NITs) nas universidades brasileiras. Liinc em Revista, 8(1), 125-140.

CK-12 Foundation. (2017). Chi Square Test. Recuperado em 30 de agosto, 2017, de https://www.ck12.org/c/statistics/chi-square-test/

Desidério, P. H. M., \& Zilber, M. A. (2014). Barreiras no Processo de Transferência Tecnológica entre Agências de Inovação e Empresas: observações em universidades públicas e privadas. Revista Gestão \& Tecnologia, 14(2), 101-126.

Howell, D. C. (2011) Chi-Square test: analysis of contingency tables. (cap. 86, pp. 250-252). Berlin: Springer-Verlag.

Instituto Nacional de Propriedade Intelectual. (2014). Inventando o futuro: uma introdução às patentes para as pequenas e médias empresas. Recuperado em 23 setembro, 2016, de http://www.inpi.gov.br/sobre/arquivos/03 cartilhapatentes $21012014 \quad 0 . p d f$

Ismail, N., Nor, M. J. M., \& Sidek, S. (2015). A framework for a successful research products commercialisation: a case of Malaysian academic researchers. Procedia Social an Behavioral Sciences, 195(Special issues), 283-292.

Johnson, D.K.N. (2002). The OECD technology concordance (OTC): Patents by industry of manufacture and sector of use. [SIT Working papers, $\mathrm{n}^{\circ}$ 5]. Paris: OCDE.

Kalar, B., \& Antoncic, B. (2015, February). The entrepreneurial university, academic activities and technology and knowledge transfer in four European countries. Technovation, 36, 1-11.

Kortum, S., \& Jonathan, P. (1997). Assigning Patents to Industries: Tests of the Yale Technology Concordance. Economic Systems Research, 9(2).

Lei n. 10.973, de 2 de dezembro de 2004 (2004). Dispõe sobre incentivos à inovação e à pesquisa científica e tecnológica ... Diário Oficial da União. Brasília, DF.

Lei n. 13.243, de 11 de janeiro de 2016 (2016). Dispõe sobre estímulos ao desenvolvimento científico, à pesquisa, à capacitação científica e tecnológica e à inovação .... Diário Oficial da República Federativa do Brasil, Brasília, DF.

Lybbert, T. J., \& Zolas, N. J. (2014) Getting patents and economic data to speak to each other: An 'algorithmic links with probabilities' approach for joint analyses of patenting and economic activity. Research Policy, 43(3), 530-542. 
Marques, R. H., Pereira, R. M., \& Garcia, M. O., G. R. (2017). Monitoramento tecnológico: um estudo de uma patente da Universidade Federal de Viçosa. Revista Gestão \& Tecnologia, 6(3), 110-137.

Mueller, S. P. M., \& Perucchi, V. (2014). Universidades e a produção de patentes: tópicos de interesse para o estudioso da informação tecnológica. Perspectivas em Ciência da Informação, 19(2), 15-36.

Nelson, R, R. (2006). The Oxford Handbook of Innovtion. New York: Oxford University Press.

Oliveira, L. G., \& Nunes, J. S. (2009). O uso do sistema de patentes pela universidade brasileira. Recuperado em 19 set., 2017, de https://digitalis.uc.pt/ptpt/ivro/o_uso_do_sistema_de_patentes_pelas_universidades_brasileiras

Organisation for Economic Co-operation and Development. (2009). OECD Patent Statistics Manual. Recuperado em 19 nov., 2017, de http://www.oecdilibrary.org/docserver/download/9209021e.pdf?expires=1505841109\&id=id\&accn ame $=$ guest $\&$ checksum $=$ C2120AC300A08F1395EB66EE94C4C93E

Pereira, F. C., \& Mello, J. M. C. (2015, outubro). Depósito de patentes de universidades brasileiras na base do INPI. Anais do 35을 Encontro Nacional de Engenharia de Produção, Fortaleza, CE, Brasil.

Perkmann, M., Tartari, V., McKelvey, M., Autio, E., Brostrom, A., D'Este, P., Fini, R., \& Geuna, A. (2013). Academic engagement and commercialization: a review of literature on university-industry relations. Research Policy, 42(2), 424-442.

Prado, M. V. B. (2012). Métodos de análise de correspondência múltipla: estudo de caso aplicado à avaliação da qualidade do café. (Dissertação de mestrado, Universidade Federal de Lavras, Lavras, MG, Brasil).

Rosa, R. A., \& Frega, J. R. (2017). Intervenientes do processo de transferência tecnológica em uma universidade pública. Revista de Administração Contemporânea, 21(4), 435-457.

Ranking Universitário da Folha. (2016). Recuperado em 19 set., 2017, de http://ruf.folha.uol.com.br/2017/

Schmoch, U., Laville, F., Patel P., \& Frietsch, R (2003). Linking Technology Areas to Industrial Sectors (Final Reports to the European Commission). Karlsrushe: DG Research.

Thursby, M., \& Gupta-Mukherjee, S. (2007). Are there real effects of licensing on academic research? A life cycle view. Journal of Economic Behavior \& Organization, 64(4).

United Nations. (2008). International Standard Industrial Classification. Recuperado em 5 jun., 2017, de https://unstats.un.org/unsd/publication/seriesM/seriesm 4rev4e.pdf 
United Nations. (2016). United Nations Statistics Division. Recuperado em 19 set., 2017, de http://unstats.un.org/unsd/cr/ctryreg/ctrydetail.asp?id=1036

Verspargen, B., Morgastel, T., \& Slabbers, M. (1994). MERIT concordance table: IPC-ISIC (rev. 2) [MERIT Research Memorandum 2/94-004]. Maastricht: University of Limburg. 